\title{
Traditional Medicines Used as Adjuvant Therapy for COVID-19 Symptoms in Syria: An Ethno-medicine Survey
}

\author{
Abdulhakim Nattouf \\ Damascus University \\ Mohamad Isam Hasan Agha \\ Damascus University
}

Chadi Khatib ( $\nabla$ chadi.khatib@gmail.com )

Damascus University https://orcid.org/0000-0002-4061-1506

Research

Keywords: Traditional Arabic Medicine (TAM), Herbal Medicine, Mediterranean, Phytotherapy, COVID-19, Folk Uses, Ethnobotanical, Ethnopharmacology.

Posted Date: March 22nd, 2021

DOI: https://doi.org/10.21203/rs.3.rs-337854/v1

License: (c) (i) This work is licensed under a Creative Commons Attribution 4.0 International License. Read Full License 


\section{Abstract}

Context: The use of traditional Arabic medicine (TAM) has spread to treat various diseases in Syria since ancient time. They are cost-effective with fewer side effects and are more suitable for long-term use compared with chemically synthesized medicines.

Objective: We conducted ethnobotanical and ethno-medicine research on plants traditionally used as adjuvant therapy for COVID-19 symptoms in Syria.

Methods: Information was collected in the period of (September 1st, 2020 to December 21st, 2020), from Southern Region (Damascus, As Suwayda, Dar'a), Northern region (especially Aleppo), Central region (Himis, Hamah), Western coastal region (Latakia, Tartus) Eastern Region (Dayr az Zawr, Al Hasakah) in Syria. 150 informants were divided into two group one of them is pharmacists who interested in herbal remedies $73.34 \%$ (63.64\% female and $36.36 \%$ male of them), and the other is herbalists $26.66 \%$ who are called "attarin" traditional healers and bee therapists. Medicinal plants being mentioned by the Informants were recorded with local names and photographed. Each reported medicinal plant species was gathered, compressed, dehydrated, and identified.

Results: In this research we listed a total of 26 medicinal species relating to 15 botanical families were generally utilized by pharmacists and herbalists in the prevention and treatment of COVID 19. The calculated results of medicinal use-value MUV showed that Pimpinella anisum L. was ranked first (MUV=0.933) followed by Zingiber officinale Roscoe. $(M U V=0.920)$, then Thymus syriacus Boiss. (MUV=0.9).

Conclusion: There may be some effective Syrian traditional herbal remedies in preventing and treating COVID-19 symptoms for some people, but the lack of information on the mechanism of effect, the appropriate dosage, side effects, toxicity and drug interactions makes them questionable, as they need more research and study.

\section{Introduction}

The World Health Organization affirms that the use of traditional medicines (TM) has a long history in maintaining health and preventing and treating diseases, especially chronic diseases. The use of Complementary \& Alternative Medicine / Traditional Medicine (CAM / TM) is an inspiration for modern pharmaceutical research. And a source of effective treatments for many diseases [1], as the organization estimates that a large proportion of the world's population depends mainly on traditional medicinal treatments for their health care, and plants have formed the basis of folk traditional medicine systems, including Ayurveda. Medicine, Greco-Arabic Medicine, Chinese Medicine and others. These systems of medicine have led to the emergence of some important medicines of natural origin that are still used today [2], as these medicines of natural origin offer an infinite variety of compounds. In chemical terms, these are often basic compounds whose structure can be utilized and the development of new compounds can be utilized by structure-to-effect relationship [3, 4]. Many common natural ingredients have been used in traditional methods of treating ailments [5].

Coronavirus (CoVs), a class of single-stranded RNA viruses, can affect both animals and humans, causing respiratory, gastrointestinal, hepatic, and neurologic diseases [6]. The first case of the novel coronavirus was reported on December 30, 2019 , in the city of Wuhan, Hubei province, P.R. China $[7,8]$.

This causative virus was temporarily named as severe acute respiratory syndrome coronavirus 2 and the resulting disease was named coronavirus disease 2019 (COVID-19) by the World Health Organization [9]. On March 11th, the WHO formally announced that COVID-19 was a pandemic [10]. COVID-19 was confirmed to have reached Syria on March 22th 2020. according to Syrian Health Ministry (http://www.moh.gov.sy/).

People with COVID-19 have had a wide range of symptoms reported - ranging from mild symptoms to severe illness. symptoms may appear 2-14 days after exposure to the virus. people with these symptoms may have COVID-19: fever or chills, cough, shortness of breath or difficulty breathing, fatigue, muscle or body aches, headache, new loss of taste or smell, sore throat, congestion or runny nose, nausea or vomiting, diarrhea [11]. Considering that viruses have become a major cause of death in

Page 2/21 
many life-threatening diseases [12], and because viruses are RNA or DNA surrounded by a nuclear envelope, these viruses have the ability to harness the molecular components of host cells to complete their cycle of replication and replication, which is They contain few targets that antiviral agents [13] can act upon, and despite the tremendous advances in chemical anti-viral drugs, there are many problems that can be encountered when using chemical treatments, including safety and efficacy limitations, as well. On the high costs $[14,15]$, it is why people have used diverse medicinal plants as alternatives which originated from ancient popular medicine. Medicinal plants continue to be used in cities and rural communities in Syria as part of healthcare, because of their low cost and the easy access to plant products in all markets across the country.

Syria is one of the nations of the Mediterranean area with diverse climatic conditions that are favorable for the development of rich and varied vegetation, and is rich in plant species. Moreover, Syrians have excellent medical knowledge and traditional experience of basic medicinal plants.

\section{Methods}

\subsection{Study area}

Syria is located on the east coast of the Mediterranean sea in southwestern Asia (Fig. 1).

\subsection{Epidemiological situation of Syria}

The study was conducted (September 1st, 2020 to December 21st, 2020), the Ministry of Health in Syria had reported that 16401 confirmed COVID-19 total cases had been reported nationally, and 10,885 recovered cases, while 1094 deaths, till 13th march 2021, according to Syrian Health Ministry (http://www.moh.gov.sy/).

\subsection{Methodology}

\subsubsection{Ethnopharmacological data collection}

A semi structured questionnaire was prepared in order to document indigenous anti-coronavirus plant use, traditional knowledge and the level of utilization of traditional medicinal plants for prevention and treatment of COVID-19. 150 informants were divided into two group one of them is pharmacists who interested in herbal remedies $73.34 \%$ (63.64\% female and $36.36 \%$ male of them), and the other is herbalists $26.66 \%$ who are called "attarin" traditional healers and bee therapists. $40 \%$ of the surveyed informants were from Southern Region (Damascus, As Suwayda, Dar'a), 20\% were from Northern region (especially Aleppo), 15\% were from Central region (Himis, Hamah), 15\% were from Western coastal region (Latakia, Tartus) and 10\% were from Eastern Region (Dayr az Zawr, Al Hasakah). A questionnaire was administered to the informants through face-to-face interviews over the period of September 1st, 2020 to December 21st, 2020, ranging from 15 to 30 min.

The inclusion criteria was that the pharmacists had interested in herbal remedies, and the herbalist had to be famous in his area. Herbalists were excluded if their experience less than 3 years. Information that had been carried to the region from the outside and that was not used or confirmed were not included and recorded

The information gathered during the present study included socio-demographic characteristics of the interviewed informants (age, gender, academic level, and experience in herbalism) and ethnopharmacological information, including the local and scientific name of the species, local names, plant parts used, modes of use, conservation method, administration mode and toxicity, all documented data were then translated into English and Latin (Appendix A).

\subsubsection{Taxonomic identification of the species}

Medicinal plants being mentioned by the Informants were recorded with local names and photographed. Each reported medicinal plant species was gathered, compressed, dehydrated, and identified, the taxonomic identity of the plants was confirmed by Prof. Abdel Aleem Bello (PhD / Botanical Taxonomy) and Dr. Bayan Tiba (PhD / Botanical Taxonomy) Aleppo university, As far as possible, the name of the plants was updated by consulting the latest literature; generic and species names followed the plant list (http://www.theplantlist.org). All voucher specimens have been preserved during documentation and deposited in the Damascus University, faculty of pharmacy, pharmacognosy labs Herbarium for future reference.

\subsubsection{Ethics approval and consent to participate}

Page $3 / 21$ 
The study was approved by the Ethics Committee of the University of Damascus. Before beginning data collection, we obtained verbal informed permission in each case site-wide and then individually before each interview. We also informed pharmacists and herbalists that it was an academic project and that the investigation was for research purposes only, and not for any financial or other benefits. Informants provided verbal informed consent to participate in this study; They were free to withdraw their information at any time. These informants freely accepted the interview.

\subsubsection{Consent for publication}

The study has a consent for publication by the faculty of pharmacy - University of Damascus on 1/3/2021 .

\subsubsection{Data analysis}

The data collected through interviews of the informants (herbalists and pharmacists) were classified and examined with the statistical program IBM ${ }^{\circledR}$ SPSS ${ }^{\circledR}$ Statistics 26 , to determine the proportions of different variables such as ethnopharmacological data. Quantitative value indices were analyzed using different statistical quantitative tools i.e. the use reports (UR) of a species, and medicinal use value (MUV). [17]

\section{Medicinal use-value (MUV) and use reports (UR)}

The use reports (UR) of a species or its importance in the culture of a community is denoted by its mentioning rate or its mention frequency by informants. The UR of the species of plants being utilized was evaluated using the formula [18]:

$\mathrm{UR}=\mathrm{Ni} / \mathrm{n}$

$\mathrm{Ni}$ : the number of times a particular species was mentioned

$\mathrm{n}$ : the total number of times that all species were mentioned

The MUV of recorded medicinal plants was determined by applying the following formula [19]:

$M U V=\sum U R i / N$

$\sum$ URi: the total number of use reports per plants

$\mathrm{N}$ : the total of interviewees questioned for given medicinal species

The MUV rate will be more important if there are several useful records for a species, implying that the plant is significant, whereas they will be near 0 if there are few reports compared to its use $[17,20]$.

\section{Results}

\subsection{Demography data of informants}

In total, 150 informants were divided into two group one of them is pharmacists who interested in herbal remedies $73.34 \%$ (63.64\% female and $36.36 \%$ male of them), and the other is herbalists $26.66 \%$ who are called "attarin" traditional healers and bee therapists. All of them were interrogated using semi-structured questionnaires. $40 \%$ of the surveyed informants were from Southern Region (Damascus, As Suwayda, Dar'a), 20\% were from Northern region (especially Aleppo), 15\% were from Central region (Himis, Hamah), 15\% were from Western coastal region (Latakia, Tartus) and 10\% were from Eastern Region (Dayr az Zawr, Al Hasakah). (Table 1). In Syria, both genders were interested in herbal medicines. So that, the ratio is close to each other, male $53.33 \%$, female $46.66 \%$ (bearing in mind that only females are pharmacists and there are no female as traditional nonspecialist herbalists).

In this study, results showed that the majority of informants interviewed were between 30 and 50 years old (95), while 33 of the informants were younger than thirty years old. and 22 of the informants were over 50 years old. Regarding the academic level, our results showed that $(40 \%)$ of the herbalists from informants had secondary education, $37.5 \%$ had primary education, and $22.5 \%$ of the herbalists had graduate education, and no one was illiterate.

Page $4 / 21$ 
Table 1

Demographic characteristics of the informants $(n=150)$.

\begin{tabular}{|llll|}
\hline Variables & Categories & Number of informants & $\%$ \\
\hline \multirow{2}{*}{ Age } & Male & 80 & 53.33 \\
\cline { 2 - 4 } & Female & 70 & 46.66 \\
& Less 30 years & 33 & 22 \\
\cline { 2 - 4 } Academic level & Between 30-50 & 95 & 63.34 \\
\cline { 2 - 4 } & Above 50 years & 22 & 14.66 \\
& Illiterate & 0 & 0 \\
\cline { 2 - 4 } & Primary & 09 & 6 \\
\cline { 2 - 4 } & Secondary & 16 & 10.6 \\
\cline { 2 - 4 } Years of experience & University & 15 & 10 \\
\cline { 2 - 4 } & Between 03-10 years & 40 & 73.4 \\
\cline { 2 - 4 } & Between 10-20 years & 60 & 26.6 \\
\cline { 2 - 4 } & Over 20 years & 50 & 40 \\
\cline { 2 - 4 } & & 110 & 33.4 \\
\hline
\end{tabular}

\subsection{Botanical families of plants used}

The present research showed that a total of 26 plant species belonging to 15 families were frequently used by pharmacists and herbalists from Syria in the prevention and treatment of COVID-19 symptoms. The family Lamiaceae was represented by the largest number of plant species (six species, 23.07\%), whereas each of Apiaceae, Myrtaceae (two species, 11.53\%), Asteraceae, and Zingiberaceae contributed with two species to each family. The remaining ten botanical families were represented by one species in each. The vernacular names, scientific names of documented species, their families, mode of preparations, used parts, MUV, were illustrated in Table 2. 
Table 2

List of plant species used to treat the symptoms of COVID-19 in the Syria.

\begin{tabular}{|c|c|c|c|c|c|c|c|}
\hline Family & $\begin{array}{l}\text { Scientific } \\
\text { name }\end{array}$ & $\begin{array}{l}\text { Common } \\
\text { name }\end{array}$ & $\begin{array}{l}\text { Part } \\
\text { used }\end{array}$ & $\begin{array}{l}\text { Mode of } \\
\text { preparation }\end{array}$ & $\begin{array}{l}\text { Bioactive } \\
\text { compounds }\end{array}$ & $\begin{array}{l}\text { Biological } \\
\text { activities }\end{array}$ & MUV \\
\hline Anacardiaceae & $\begin{array}{l}\text { Rhus } \\
\text { aromatica } \\
\text { Aiton }\end{array}$ & 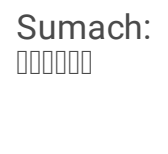 & Fruits & Decoction & $\begin{array}{l}\text { Volatile Oil, Phenol } \\
\text { Glycosides, } \\
\text { Steroids, Tannins, } \\
\text { Triterpenes [21] }\end{array}$ & $\begin{array}{l}\text { Antiviral, } \\
\text { Antimicrobial } \\
{[22,23]}\end{array}$ & 0.333 \\
\hline \multirow[t]{6}{*}{ Apiaceae } & \multirow[t]{2}{*}{$\begin{array}{l}\text { Pimpinella } \\
\text { anisum L. }\end{array}$} & \multirow[t]{2}{*}{$\begin{array}{l}\text { Yansoon: } \\
\text { प्याप्रा }\end{array}$} & \multirow[t]{2}{*}{ Seeds } & \multirow[t]{2}{*}{ Infusion } & \multirow[t]{2}{*}{$\begin{array}{l}\text { Volatile Oil, Caffeic } \\
\text { Acid Derivatives, } \\
\text { Flavonoids, Fatty } \\
\text { Oil, Proteic } \\
\text { Substances [21] }\end{array}$} & $\begin{array}{l}\text { Anti- } \\
\text { Inflammatory, } \\
\text { Antioxidant, } \\
\text { Antiviral, } \\
\text { Antibacterial, } \\
\text { Anticancer }\end{array}$ & \multirow[t]{2}{*}{0.933} \\
\hline & & & & & & {$[24,25,26]$} & \\
\hline & \multirow[t]{2}{*}{$\begin{array}{l}\text { Foeniculum } \\
\text { vulgare Mill. }\end{array}$} & \multirow[t]{2}{*}{$\begin{array}{l}\text { Shamra: } \\
\text { पिए }\end{array}$} & \multirow[t]{2}{*}{ Seeds } & \multirow[t]{2}{*}{ Infusion } & \multirow[t]{2}{*}{$\begin{array}{l}\text { Volatile Oil, } \\
\text { Hydroxycoumarins, } \\
\text { Pyranocoumarins, } \\
\text { Flavonoids, Fatty } \\
\text { Oil [21] }\end{array}$} & $\begin{array}{l}\text { Antioxidant, } \\
\text { Anti- } \\
\text { Inflammatory, } \\
\text { Antiviral, } \\
\text { Anticancer }\end{array}$ & \multirow[t]{2}{*}{0.233} \\
\hline & & & & & & $\begin{array}{l}{[27,28,29,} \\
30]\end{array}$ & \\
\hline & \multirow[t]{2}{*}{$\begin{array}{l}\text { Coriandrum } \\
\text { sativum L. }\end{array}$} & \multirow[t]{2}{*}{$\begin{array}{l}\text { Kozbara: } \\
\text { स०स्य }\end{array}$} & \multirow[t]{2}{*}{$\begin{array}{l}\text { Fruits, } \\
\text { Seeds }\end{array}$} & \multirow[t]{2}{*}{ Infusion } & \multirow[t]{2}{*}{$\begin{array}{l}\text { Volatile Oil, Fatty } \\
\text { Oil, } \\
\text { Hydroxycoumarins } \\
{[21]}\end{array}$} & $\begin{array}{l}\text { Antimicrobial, } \\
\text { Antiviral, } \\
\text { Antioxidant }\end{array}$ & \multirow[t]{2}{*}{0.133} \\
\hline & & & & & & {$[31,32,33]$} & \\
\hline \multirow[t]{2}{*}{ Aquifoliaceae } & \multirow[t]{2}{*}{$\begin{array}{l}\text { Ilex } \\
\text { paraguariensis } \\
\text { A.St.-Hil. }\end{array}$} & \multirow[t]{2}{*}{ Mate: } & \multirow[t]{2}{*}{ Leaves } & \multirow[t]{2}{*}{ Decoction } & \multirow{2}{*}{$\begin{array}{l}\text { Volatile Oil, Purine } \\
\text { Alkaloids, Caffeic } \\
\text { Acid Derivatives, } \\
\text { Flavonoids, } \\
\text { Triterpene } \\
\text { Saponins, Nitrite } \\
\text { Glycosides [21] }\end{array}$} & $\begin{array}{l}\text { Antimicrobial, } \\
\text { Antiviral, } \\
\text { Antioxidant, } \\
\text { Anticancer }\end{array}$ & \multirow[t]{2}{*}{0.266} \\
\hline & & & & & & $\begin{array}{l}{[34,35,36,} \\
37]\end{array}$ & \\
\hline \multirow[t]{2}{*}{ Araliaceae } & \multirow[t]{2}{*}{$\begin{array}{l}\text { Panax ginseng } \\
\text { C.A.Mey. }\end{array}$} & \multirow[t]{2}{*}{ 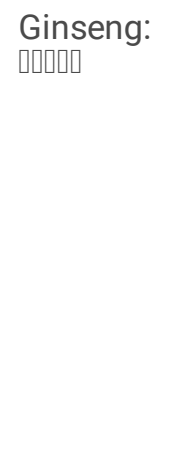 } & \multirow[t]{2}{*}{ Roots } & \multirow[t]{2}{*}{$\begin{array}{l}\text { Powder, } \\
\text { Decoction }\end{array}$} & \multirow[t]{2}{*}{$\begin{array}{l}\text { Triterpene } \\
\text { Saponins, } \\
\text { Polysaccharides, } \\
\text { Polyynes [21] }\end{array}$} & $\begin{array}{l}\text { Antimicrobial, } \\
\text { Antiviral, } \\
\text { Antioxidant, It } \\
\text { Has Expected } \\
\text { Potential } \\
\text { Against } \\
\text { COVID-19, } \\
\text { Anti SARS- } \\
\text { Cov-2 } \\
\text { Infection }\end{array}$ & \multirow[t]{2}{*}{0.8} \\
\hline & & & & & & $\begin{array}{l}{[38,39,40,} \\
41]\end{array}$ & \\
\hline \multirow[t]{2}{*}{ Asteraceae } & \multirow[t]{2}{*}{$\begin{array}{l}\text { Artemisia } \\
\text { herba-alba } \\
\text { Asso }\end{array}$} & \multirow[t]{2}{*}{ Chih:णाया } & \multirow[t]{2}{*}{ Leaf } & \multirow[t]{2}{*}{ Decoction } & $\begin{array}{l}\text { Volatile Oil, } \\
\text { Sesquiterpene } \\
\text { Lactones, } \\
\text { Lipophilic } \\
\text { Flavonoids, } \\
\text { Polyynes, } \\
\text { Hydroxycoumarins } \\
\text { [21] }\end{array}$ & $\begin{array}{l}\text { It Has } \\
\text { Expected } \\
\text { Potential } \\
\text { Against } \\
\text { COVID-19, } \\
\text { Anti SARS- } \\
\text { Cov-2 } \\
\text { Infection, } \\
\text { Antimalarial, } \\
\text { Antioxidant, } \\
\text { Antibacterial, }\end{array}$ & 0.733 \\
\hline & & & & & & $\begin{array}{l}{[42,43,44,} \\
45,46]\end{array}$ & \\
\hline
\end{tabular}




\begin{tabular}{|c|c|c|c|c|c|c|c|}
\hline Family & $\begin{array}{l}\text { Scientific } \\
\text { name }\end{array}$ & $\begin{array}{l}\text { Common } \\
\text { name }\end{array}$ & $\begin{array}{l}\text { Part } \\
\text { used }\end{array}$ & $\begin{array}{l}\text { Mode of } \\
\text { preparation }\end{array}$ & $\begin{array}{l}\text { Bioactive } \\
\text { compounds }\end{array}$ & $\begin{array}{l}\text { Biological } \\
\text { activities }\end{array}$ & MUV \\
\hline & $\begin{array}{l}\text { Matricaria } \\
\text { chamomilla L. }\end{array}$ & 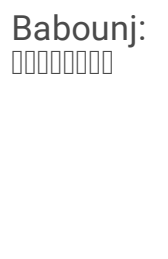 & Flowers & Infusion & $\begin{array}{l}\text { Volatile Oil, } \\
\text { Flavonoids, } \\
\text { Unbound Highly } \\
\text { Methoxylized } \\
\text { Flavonoids, } \\
\text { Hydroxycoumarins, } \\
\text { Mucilages [21] }\end{array}$ & $\begin{array}{l}\text { Antiviral, } \\
\text { Antimicrobial, } \\
\text { Anticancer, } \\
\text { Antioxidant } \\
\text { [47, 48, 49, } \\
50]\end{array}$ & 0.7 \\
\hline Fabaceae & $\begin{array}{l}\text { Glycyrrhiza } \\
\text { glabra L. }\end{array}$ & $\begin{array}{l}\text { Arq Sous: } \\
\text { परिए। }\end{array}$ & Rhizome & Decoction & $\begin{array}{l}\text { Volatile Oil, } \\
\text { Triterpene } \\
\text { Saponins, } \\
\text { Flavonoids, } \\
\text { Isoflavonoids, } \\
\text { Cumestan } \\
\text { Derivatives, } \\
\text { Hydroxycoumarins, } \\
\text { Steroids [21] }\end{array}$ & $\begin{array}{l}\text { Anti- } \\
\text { Inflammatory, } \\
\text { Antimicrobial, } \\
\text { Antioxidant, } \\
\text { Antiviral, } \\
{[51,52,53]}\end{array}$ & 0.266 \\
\hline \multirow[t]{8}{*}{ Lamiaceae } & $\begin{array}{l}\text { Melissa } \\
\text { officinalis L. }\end{array}$ & $\begin{array}{l}\text { Malissa: } \\
\text { पिए }\end{array}$ & $\begin{array}{l}\text { Leaves, } \\
\text { Flowers }\end{array}$ & Infusion & $\begin{array}{l}\text { Volatile Oil, Phenol } \\
\text { Glycosides, Caffeic } \\
\text { Acid Derivatives, } \\
\text { Flavonoids, } \\
\text { Triterpene Acids } \\
\text { [21] }\end{array}$ & $\begin{array}{l}\text { Antiviral, } \\
\text { Antimicrobial, } \\
\text { Antioxidant } \\
{[54,55,56,} \\
57]\end{array}$ & 0.333 \\
\hline & $\begin{array}{l}\text { Mentha } \\
\text { pulegium L. }\end{array}$ & 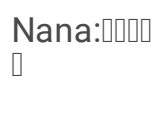 & Leaves & Infusion & $\begin{array}{l}\text { Volatile Oil, } \\
\text { Tannins, } \\
\text { Flavonoids [21] }\end{array}$ & $\begin{array}{l}\text { Antiviral, } \\
\text { Antimicrobial, } \\
\text { Antioxidant }\end{array}$ & 0.533 \\
\hline & & & & & & {$[58,59,60]$} & \\
\hline & $\begin{array}{l}\text { Micromeria } \\
\text { myrtifolia } \\
\text { Boiss. \& } \\
\text { Hohen }\end{array}$ & Zofa:स्या & $\begin{array}{l}\text { Aerial } \\
\text { Parts }\end{array}$ & Infusion & $\begin{array}{l}\text { Volatile Oil, } \\
\text { Tannins, Bitter } \\
\text { Principles, } \\
\text { Flavonoids [21] }\end{array}$ & $\begin{array}{l}\text { Antiviral, } \\
\text { Antimicrobial, } \\
\text { Antioxidant, } \\
\text { Anti- } \\
\text { Inflammatory }\end{array}$ & 0.6 \\
\hline & & & & & & {$[61,62,63]$} & \\
\hline & $\begin{array}{l}\text { Origanum } \\
\text { syriacum L. }\end{array}$ & Zaoba:[प] & $\begin{array}{l}\text { Aerial } \\
\text { Parts }\end{array}$ & Infusion & $\begin{array}{l}\text { Volatile Oil, } \\
\text { Flavonoids, Caffeic } \\
\text { Acid Derivatives } \\
\text { [21] }\end{array}$ & $\begin{array}{l}\text { Antiviral, } \\
\text { Antimicrobial, } \\
\text { Antioxidant } \\
{[64,65,66,} \\
67]\end{array}$ & 0.4 \\
\hline & $\begin{array}{l}\text { Rosmarinus } \\
\text { officinalis L. }\end{array}$ & 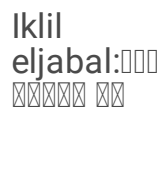 & Leaf & Infusion & $\begin{array}{l}\text { Volatile Oil, Caffeic } \\
\text { Acid Derivatives, } \\
\text { Diterpenes (Bitter), } \\
\text { Flavonoids, } \\
\text { Triterpenes [21] }\end{array}$ & $\begin{array}{l}\text { Antiviral, } \\
\text { Antimicrobial, } \\
\text { Antioxidant, } \\
{[68,69,70]}\end{array}$ & 0.466 \\
\hline & $\begin{array}{l}\text { Thymus } \\
\text { syriacus } \\
\text { Boiss. }\end{array}$ & $\begin{array}{l}\text { Zaatar: } \\
\text { क्साप्रा }\end{array}$ & $\begin{array}{l}\text { Aerial } \\
\text { Parts }\end{array}$ & Infusion & $\begin{array}{l}\text { Volatile Oil, } \\
\text { Flavonoids, Caffeic } \\
\text { Acid Derivatives } \\
\text { [21] }\end{array}$ & $\begin{array}{l}\text { Antimicrobial, } \\
\text { Antioxidant, } \\
\text { Antimicrobial } \\
{[71,72,73]}\end{array}$ & 0.9 \\
\hline
\end{tabular}




\begin{tabular}{|c|c|c|c|c|c|c|c|}
\hline Family & $\begin{array}{l}\text { Scientific } \\
\text { name }\end{array}$ & $\begin{array}{l}\text { Common } \\
\text { name }\end{array}$ & $\begin{array}{l}\text { Part } \\
\text { used }\end{array}$ & $\begin{array}{l}\text { Mode of } \\
\text { preparation }\end{array}$ & $\begin{array}{l}\text { Bioactive } \\
\text { compounds }\end{array}$ & $\begin{array}{l}\text { Biological } \\
\text { activities }\end{array}$ & MUV \\
\hline \multirow[t]{2}{*}{ Lauraceae } & \multirow[t]{2}{*}{$\begin{array}{l}\text { Cinnamomum } \\
\text { cassia (L.) } \\
\text { J.Presl }\end{array}$} & \multirow[t]{2}{*}{ 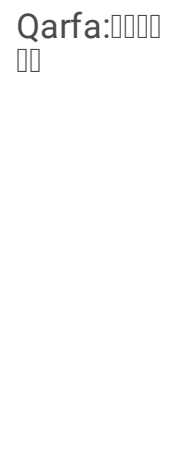 } & \multirow[t]{2}{*}{ Bark } & \multirow[t]{2}{*}{ Powder } & \multirow[t]{2}{*}{$\begin{array}{l}\text { Volatile Oil, } \\
\text { Diterpenes, } \\
\text { Tannins, Oligomere } \\
\text { Proanthocyanidins, } \\
\text { Mucilages [21] }\end{array}$} & $\begin{array}{l}\text { It Has } \\
\text { Expected } \\
\text { Potential } \\
\text { Against } \\
\text { COVID-19, } \\
\text { Anti SARS- } \\
\text { Cov-2 } \\
\text { Infection, } \\
\text { Antimicrobial, } \\
\text { Antioxidant }\end{array}$ & \multirow[t]{2}{*}{0.2} \\
\hline & & & & & & $\begin{array}{l}{[74,75,76,} \\
77]\end{array}$ & \\
\hline \multirow[t]{6}{*}{ Myrtaceae } & \multirow[t]{2}{*}{$\begin{array}{l}\text { Eucalyptus } \\
\text { globulus } \\
\text { Labill. }\end{array}$} & \multirow[t]{2}{*}{ 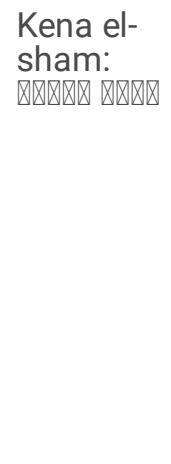 } & \multirow[t]{2}{*}{ Leaves } & \multirow[t]{2}{*}{$\begin{array}{l}\text { Infusion } \\
\text { For } \\
\text { Inhalation }\end{array}$} & \multirow[t]{2}{*}{$\begin{array}{l}\text { Volatile Oil, } \\
\text { Flavonoids [21] }\end{array}$} & $\begin{array}{l}\text { It Has } \\
\text { Expected } \\
\text { Potential } \\
\text { Against } \\
\text { COVID-19, } \\
\text { Anti SARS- } \\
\text { Cov-2 } \\
\text { Infection, } \\
\text { Antimicrobial, } \\
\text { Antioxidant }\end{array}$ & \multirow[t]{2}{*}{0.4} \\
\hline & & & & & & $\begin{array}{l}{[78,79,80,} \\
81]\end{array}$ & \\
\hline & \multirow[t]{2}{*}{$\begin{array}{l}\text { Myrtus } \\
\text { communis L. }\end{array}$} & \multirow[t]{2}{*}{ Aas:वायु } & \multirow[t]{2}{*}{$\begin{array}{l}\text { Fruits, } \\
\text { Leaves }\end{array}$} & \multirow[t]{2}{*}{ Infusion } & \multirow[t]{2}{*}{$\begin{array}{l}\text { Volatile Oil, } \\
\text { Tannins, } \\
\text { Acylphloroglucinols } \\
{[21]}\end{array}$} & $\begin{array}{l}\text { It Has } \\
\text { Expected } \\
\text { Potential } \\
\text { Against } \\
\text { COVID-19, } \\
\text { Anti SARS- } \\
\text { Cov-2 } \\
\text { Infection, } \\
\text { Antimicrobial, } \\
\text { Antioxidant }\end{array}$ & 0.266 \\
\hline & & & & & & $\begin{array}{l}{[82,83,84,} \\
85]\end{array}$ & \\
\hline & \multirow[t]{2}{*}{$\begin{array}{l}\text { Eugenia } \\
\text { caryophyllata } \\
\text { Thunb. }\end{array}$} & \multirow[t]{2}{*}{$\begin{array}{l}\text { Qronfel: } \\
\text { वसास्या }\end{array}$} & \multirow[t]{2}{*}{$\begin{array}{l}\text { Aromatic } \\
\text { Flower } \\
\text { Buds }\end{array}$} & \multirow[t]{2}{*}{ Maceration } & \multirow[t]{2}{*}{$\begin{array}{l}\text { Volatile Oil, } \\
\text { Flavonoids, } \\
\text { Tannins, } \\
\text { Triterpenes, } \\
\text { Steroids [21] }\end{array}$} & $\begin{array}{l}\text { Anti- } \\
\text { Inflammatory, } \\
\text { Antimicrobial, } \\
\text { Antioxidant }\end{array}$ & 0.366 \\
\hline & & & & & & {$[86,87,88]$} & \\
\hline \multirow[t]{2}{*}{ Oleaceae } & \multirow[t]{2}{*}{$\begin{array}{l}\text { Olea europaea } \\
\text { L. }\end{array}$} & \multirow[t]{2}{*}{ 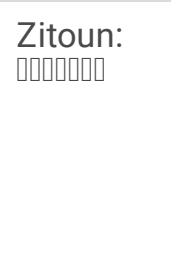 } & \multirow[t]{2}{*}{$\begin{array}{l}\text { Leaf, } \\
\text { Fruits }\end{array}$} & \multirow[t]{2}{*}{$\begin{array}{l}\text { Decoction, } \\
\text { Oil }\end{array}$} & \multirow{2}{*}{$\begin{array}{l}\text { Flavonoids, } \\
\text { Flavone } \\
\text { Glycosides, } \\
\text { Flavanones, } \\
\text { Iridoids, } \\
\text { Triterpenes, } \\
\text { Biophenols [21] }\end{array}$} & $\begin{array}{l}\text { Antioxidant, } \\
\text { Antiviral, } \\
\text { Antimicrobial }\end{array}$ & 0.166 \\
\hline & & & & & & {$[89,90,91]$} & \\
\hline \multirow[t]{2}{*}{ Ranunculaceae } & \multirow[t]{2}{*}{$\begin{array}{l}\text { Nigella sativa } \\
\text { L. }\end{array}$} & \multirow[t]{2}{*}{ 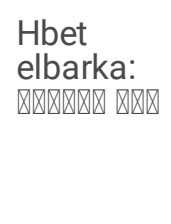 } & \multirow[t]{2}{*}{ Seed } & \multirow[t]{2}{*}{ Powder, Oil } & \multirow[t]{2}{*}{$\begin{array}{l}\text { Nigellidine, } \\
\text { Nigellicine, } \\
\text { Carvacrol, Thymol, } \\
\text { A- Hederin [92] }\end{array}$} & $\begin{array}{l}\text { Anti- } \\
\text { Inflammatory, } \\
\text { Antiviral, } \\
\text { Antimicrobial }\end{array}$ & 0.66 \\
\hline & & & & & & {$[93,94,95]$} & \\
\hline
\end{tabular}




\begin{tabular}{|c|c|c|c|c|c|c|c|}
\hline Family & $\begin{array}{l}\text { Scientific } \\
\text { name }\end{array}$ & $\begin{array}{l}\text { Common } \\
\text { name }\end{array}$ & $\begin{array}{l}\text { Part } \\
\text { used }\end{array}$ & $\begin{array}{l}\text { Mode of } \\
\text { preparation }\end{array}$ & $\begin{array}{l}\text { Bioactive } \\
\text { compounds }\end{array}$ & $\begin{array}{l}\text { Biological } \\
\text { activities }\end{array}$ & MUV \\
\hline \multirow[t]{2}{*}{ Rosaceae } & \multirow[t]{2}{*}{$\begin{array}{l}\text { Rosa } \\
\text { xdamascena } \\
\text { Mill. }\end{array}$} & \multirow[t]{2}{*}{ 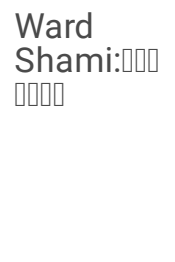 } & \multirow[t]{2}{*}{ Petals } & \multirow[t]{2}{*}{$\begin{array}{l}\text { Infusion, } \\
\text { Syrup }\end{array}$} & \multirow[t]{2}{*}{$\begin{array}{l}\text { Volatile Oil, } \\
\text { Flavonol } \\
\text { Glycosides }[96,97]\end{array}$} & $\begin{array}{l}\text { Anti- } \\
\text { Inflammatory, } \\
\text { Antioxidant, } \\
\text { Antimicrobial }\end{array}$ & \multirow[t]{2}{*}{0.533} \\
\hline & & & & & & $\begin{array}{l}{[98,99,100,} \\
101]\end{array}$ & \\
\hline \multirow[t]{2}{*}{ Rutaceae } & \multirow[t]{2}{*}{$\begin{array}{l}\text { Citrus limon } \\
\text { (L.) Osbeck }\end{array}$} & \multirow[t]{2}{*}{ 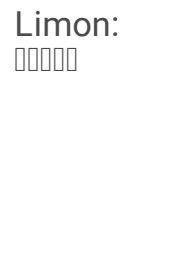 } & \multirow[t]{2}{*}{ Fruit } & \multirow[t]{2}{*}{ Maceration } & \multirow[t]{2}{*}{$\begin{array}{l}\text { Volatile Oil, } \\
\text { Flavonoids, } \\
\text { Vitamin C [21] }\end{array}$} & $\begin{array}{l}\text { Anti- } \\
\text { Inflammatory, } \\
\text { Antioxidant, } \\
\text { Antimicrobial }\end{array}$ & \multirow[t]{2}{*}{0.633} \\
\hline & & & & & & $\begin{array}{l}{[102,103,} \\
104]\end{array}$ & \\
\hline \multirow[t]{2}{*}{ Solanaceae } & \multirow[t]{2}{*}{$\begin{array}{l}\text { Capsicum } \\
\text { annuum L. }\end{array}$} & \multirow[t]{2}{*}{ 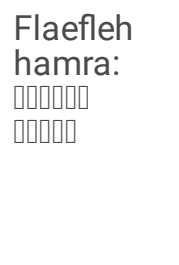 } & \multirow[t]{2}{*}{ Fruit } & \multirow[t]{2}{*}{ Powder } & \multirow[t]{2}{*}{$\begin{array}{l}\text { Volatile Oil, } \\
\text { Capsaicinoids, } \\
\text { Carotinoids, } \\
\text { Flavonoids, Steroid } \\
\text { Saponins [21] }\end{array}$} & $\begin{array}{l}\text { Anti- } \\
\text { Inflammatory, } \\
\text { Antioxidant, } \\
\text { Antimicrobial }\end{array}$ & \multirow[t]{2}{*}{0.2} \\
\hline & & & & & & $\begin{array}{l}{[105,106,} \\
107]\end{array}$ & \\
\hline \multirow[t]{4}{*}{ Zingiberaceae } & \multirow[t]{2}{*}{$\begin{array}{l}\text { Curcuma } \\
\text { longa L. }\end{array}$} & \multirow[t]{2}{*}{$\begin{array}{l}\text { Curcum: } \\
\text { [uा] }\end{array}$} & \multirow[t]{2}{*}{ Rhizome } & \multirow[t]{2}{*}{ Powder } & \multirow[t]{2}{*}{$\begin{array}{l}\text { Volatile Oil, } \\
\text { Curcuminoids, 1,5- } \\
\text { Diaryl-Penta-1,4- } \\
\text { Dien-3-One } \\
\text { Derivatives [21] }\end{array}$} & $\begin{array}{l}\text { Anti- } \\
\text { Inflammatory, } \\
\text { Antioxidant, } \\
\text { Antimicrobial }\end{array}$ & \multirow[t]{2}{*}{0.4} \\
\hline & & & & & & $\begin{array}{l}{[108,109} \\
110,111]\end{array}$ & \\
\hline & \multirow[t]{2}{*}{$\begin{array}{l}\text { Zingiber } \\
\text { officinale } \\
\text { Roscoe }\end{array}$} & \multirow[t]{2}{*}{ 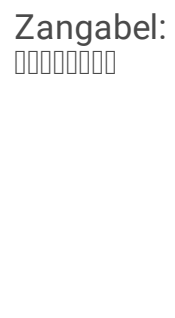 } & \multirow[t]{2}{*}{$\begin{array}{l}\text { Rhizome, } \\
\text { Root }\end{array}$} & \multirow[t]{2}{*}{$\begin{array}{l}\text { Powder, } \\
\text { Fresh }\end{array}$} & \multirow[t]{2}{*}{$\begin{array}{l}\text { Volatile Oil, } \\
\text { Sesquiterpene } \\
\text { Bitter Principles } \\
\text { [21] }\end{array}$} & $\begin{array}{l}\text { Anti- } \\
\text { Inflammatory, } \\
\text { Antioxidant, } \\
\text { Antimicrobial, } \\
\text { Antiviral }\end{array}$ & \multirow[t]{2}{*}{0.92} \\
\hline & & & & & & $\begin{array}{l}{[112,113,} \\
114,115, \\
116]\end{array}$ & \\
\hline
\end{tabular}

\subsection{Medicinal use plants (MUV)}

Medicinal use plants (MUV) are utilized to find the most frequently used plant species in the study area. Its value ranged from 0.133 to 0.933 (Table 2). The calculated results of MUV showed that Pimpinella anisum L. was ranked first (MUV $=0.933$ ) followed by Zingiber officinale Roscoe. (MUV = 0.920), Thymus syriacus Boiss. (MUV = 0.9), then Panax ginseng C.A.Mey. (MUV = 0.8), Artemisia herba-alba Asso (MUV = 0.733), Matricaria chamomilla L. (MUV = 0.7), Nigella sativa L. (MUV = 0.66), Citrus limon (L.) Osbeck (MUV = 0.633), Micromeria myrtifolia Boiss. \& Hohen (MUV =0.6), Mentha pulegium L. and Rosa xdamascena Mill. (MUV = 0.533), Rosmarinus officinalis L. (MUV = 0.466), Origanum syriacum L. and Eucalyptus globulus Labill. and Curcuma longa L. (MUV = 0.4), Eugenia caryophyllata Thunb. (MUV = 0.4), Melissa officinalis L. and Rhus aromatica Aiton (MUV $=0.4$ ), Ilex paraguariensis A.St.-Hil. and Glycyrrhiza glabra L. and Myrtus communis L. (MUV = 0.266), Cinnamomum cassia (L.) J.Presl and Capsicum annuum L. (MUV = 0.2), Olea europaea L. (MUV=0.166), while the lowest value was found for Coriandrum sativum $\mathrm{L}$. (MUV = 0.133).

\subsection{Parts of the plant used}

In our study area, different plant parts harvest for the preparation of herbal remedies (e.g., aerial parts, seed, rhizome, flower, and leaf). The interview results revealed that leaves were the most frequently used part of the medicinal plants $25.8 \%$ of the total, 
followed by the fruit (19.35\%), rhizome (16.12\%), each of seed and flower (12.9\%), aerial parts (9.6\%), and bark (3.22\%) (Table 2).

\subsection{Modes and conditions of medicine preparation}

In Syria, the most of the herbal recipes (81.3\%) were made from fresh material followed by their dried form (18.7\%). The recipes in the most cases were obtained from single herb, but some of recipes were prepared together, and there is a famous local mixture called Damask tisane (zhourate Shamieh) or (Syrian zahraa) [117]. The major solvent was water (59\%), honey (22\%), seeds oil (22\%) and vinegar (2\%). Herbalists and pharmacists advise various methods of preparation for COVID-19 symptoms. A mode of traditional medicine preparation reported was infusion (41.19\%), followed by decoction and powder (19.35\%), then maceration and oil (6.45\%). (Table 2).

\subsection{Symptoms, and treatment of COVID-19}

The pharmacists explained that most of the complaints from people not diagnosed with an actual infection with Covid-19 were asking to obtain vitamin $\mathrm{C}$, effervescent tablets and capsules of the mineral zinc as a dietary supplement, vitamin D in addition to propolis solution and sugar lozenges of propolis and menthol, in addition to some of the herbs mentioned in (Table 2), in order to improve their immunity. While many patients prefer to use herbs only to improve common COVID-19 symptoms which include fever, tiredness, sore throat, loss of taste and smell, headache, and dry cough.

While herbalists (40) confirmed that most of their visitors relied on natural healing, and they requested the herbs mentioned in (Table 2), in addition to different types of honey and propolis. Symptoms, they said, were improved within a week of using the herbs. In any case, this information is considered insufficient, and it needs study and clinical evaluation, but it is used by many who had symptoms similar to those of Covid-19 but did not confirm infection with labs analysis, and they relied on selfmedication. Both of pharmacists and herbalists reported that the herbal remedies shortened the duration of fever and common cold by a few days and symptoms of fatigue.

\section{Discussion}

Many countries have inherited traditional customs for their peoples in the field of treatment and hospitalization from diseases and pain, and these norms have their roots in the depths of the culture and history of these peoples. Some of these customs or therapeutic forms are in the field of traditional medicine [1].

Traditional medicine (TM), as defined by the World Health Organization (WHO), is the sum total of the knowledge, skills, and practices based on the theories, beliefs, and experiences indigenous to different cultures, whether explicable or not, used in the maintenance of health as well as in the prevention, diagnosis, improvement, or treatment of physical and mental illness. Some traditional medicine systems are supported by huge volumes of literature and records of the theoretical concepts and practical skills; others pass down from generation to generation through verbal teaching. To date, in some parts of the world, the majority of the population continue to rely on their own traditional medicine to meet their primary health care needs. When adopted outside of its traditional culture, traditional medicine is often referred as "complementary and alternative medicine (CAM)" [118].

Where the first records of traditional medicine, such as cedar oils, cypress, licorice, dates and poppy oils, date back to $2600 \mathrm{BC}$ and are still used today, and $80 \%$ of the world's population depends on traditional medicine based on Plants, as compounds derived from plant species form the basis of $50 \%$ of modern medicinal prescriptions. Therefore, the knowledge and traditions of indigenous and local communities must be preserved [119].

Herbal products are a source of interest for scientists to research the development of new drugs for many diseases. COVID-19, caused by the novel coronavirus (SARS-CoV-2), is a deadly infectious disease that has claimed many lives so far. Medicinal plants are the first choice for traditional healers in many societies to prevent and treat many diseases and ailments. Since the outbreak of the COVID-19 pandemic, many traditional herbal medicines have been used, and some of them have given good results among COVID-19 patients, especially those who had symptoms and did not confirm laboratory tests, especially in Syria. 
In this study a semi structured questionnaire was prepared in order to document indigenous anti-coronavirus plant use in Syria, traditional knowledge and the level of utilization of traditional medicinal plants for prevention and treatment of COVID-19. 150 informants were divided into two group one of them is pharmacists who interested in herbal remedies $73.34 \%$ (63.64\% female and $36.36 \%$ male of them), and the other is herbalists $26.66 \%$ who are called "attarin" traditional healers and bee therapists. $40 \%$ of the surveyed informants were from Southern Region (Damascus, As Suwayda, Dar'a), 20\% were from Northern region (especially Aleppo), 15\% were from Central region (Himis, Hamah), 15\% were from Western coastal region (Latakia, Tartus) and 10\% were from Eastern Region (Dayr az Zawr, Al Hasakah), according the result both genders are interested in herbal medicines. However, the numbers of female pharmacists participants were higher than those of males. while the herbalists as Men were predominantly represented in the sample because of their work. Females were rarely found at herbal markets as herbalists during our interviews. Regarding the age groups, results showed that the majority of informants interviewed were between 30 and 50 years old (95), while 33 of the informants were younger than thirty years old. and 22 of the informants were over 50 years old. Regarding the academic level, our results showed that $(40 \%)$ of the herbalists from informants had secondary education, $37.5 \%$ had primary education, and $22.5 \%$ of the herbalists had graduate education, and no one was illiterate.

The present study revealed that a total of 26 medicinal species relating to 15 botanical families were generally utilized by pharmacists and herbalists in the prevention and treatment of COVID 19. The family Lamiaceae was represented by the largest number of plant species (six species, 23.07\%). The calculated results of MUV showed that Pimpinella anisum L. was ranked first (MUV = 0.933) followed by Zingiber officinale Roscoe. (MUV = 0.920), Thymus syriacus Boiss. $(M U V=0.9)$, then Panax ginseng C.A.Mey. (MUV = 0.8). These medicinal species having high MUV must be further assessed for phytochemical, pharmacological, and toxicological studies to identify their active constituents for an effective and non-toxic drug extraction.

The interview results revealed that leaves are the most frequently used part of the medicinal plants $25.8 \%$ of the total, followed by the fruits $19.35 \%$, and the seeds $12.9 \%$. The choice of leaves was due to its easy availability, collection, and it is traditionally used in drug preparation. The most of the herbal recipes (81.3\%) were made from fresh material followed by their dried form $(18.7 \%)$. The recipes in the most cases were obtained from single herb, but some of recipes were prepared together, and there is a famous local mixture called Damask tisane (zhourate Shamieh) or (Syrian zahraa). The major solvent was water (59\%), honey (22\%), seeds oil (22\%) and vinegar (2\%). Herbalists and pharmacists advise various methods of preparation for COVID-19 symptoms. A mode of traditional medicine preparation reported was infusion (41.19\%), followed by decoction and powder (19.35\%), then maceration and oil (6.45\%). The frequent employment of the infusion because of it's easy-to-prepare and popular traditional method used in Syria to prepare aromatic medicinal herbs and it is more suitable for aromatic plants due to avoid the excessive heat to maintain an aromatic and volatile oils.

Popular and traditional drinks spread in Syria very widely, such as mate with aromatic medicinal herbs (hot drink as decoction), julep and Damask rose syrup (cold drink as maceration), licorice syrup (cold drink as maceration) and tamarind (cold drink as maceration), which may have an important effect in curbing infection with viral and bacterial diseases that affecting the respiratory system.

These medicinal plants contain a wide variety of bioactive compounds including volatile oil, phenol glycosides, flavonoids, fatty oils, hydroxycoumarins, triterpenes, tannins, steroids, mucilages, and organic acids. Many of these effective compounds in the studied medicinal plants have antiviral effects, especially those that contain anthocyanates, which are colored phenols found in many colorful plants and fruits. As many studies have shown its great role as an antioxidant and in delaying aging [120,121]. Scientific evidence for the role of these compounds in suppressing infections and inhibiting the growth of germs [122] has been proven. The study of the anti-viral effect of antocyanates had a large place in global research, as it showed its inhibitory effects on many stages of the virus's life, which led to its suggestion as effective treatments for viral infections[123]. And the other flavonoid derivatives have also been reported to possess antiviral activity against a wide range of viruses such as HSV, HIV, Coxsackie B virus, coronavirus, cytomegalovirus, poliomyelitis virus, rhinovirus, rotavirus, poliovirus, and rabies virus [17, 124 , 125]. Phenolic compounds, polyphenols, steroids, terpenoids, other active phytochemicals, and their derivatives are common plant secondary metabolites that contain aromatic rings with 1 or several hydroxyl groups. For a wide range of viruses, several studies confirmed the abovementioned activity of several phenolic antiviral compounds such as curcumin, luteolin-7glucoside, epicatechin gallate, catechin, demethoxycurcumin, bavachinin, apigenin-7 glucoside, silvestrol, hypericin, psoralidin,

Page 11/21 
mycophenolate mofetil, corylifol, and tomentin $[17,126,127,128]$. Also polyphenols are capable of binding easily with the lipoproteins of the virus envelope, which can prevent the viral invasion in host cells $[17,129]$.

Volatile and aromatic oils (anethole, chamazulene, limonene, thymol, eucalyptol, verbenone, $\mathrm{\gamma}$-terpinene, sabinene, caryophyllene, linalool, myrcene, camphor, camphene, borneol, menthol, eugenol, carvacrol, myrtenol, etc.) from many medicinal plants such as Origanum syriacum L., Micromeria myrtifolia Boiss. \& Hohen, Mentha pulegium L., Melissa officinalis L., Artemisia annua L., Rosmarinus officinalis L., Rosa xdamascena Mill., Pimpinella anisum L., Citrus limon (L.) Osbeck, Eugenia caryophyllata Thunb., Coriandrum sativum L., and other aromatic plants with antiviral activities were well documented by several researchers [17, 129, 130, 131, 132]. Eucalyptol, a vital essential oil from Eucalyptus globulus Labill. was identified as an effective antiviral compound against coronavirus, especially COVID-19 because this major component of eucalyptus oil consists of ether $(-0)$, ketone $(=0)$, and hydroxyl $(-\mathrm{OH})$ groups which play the main inhibitory role against SARS-CoV-2 [133]. Essential oils can insert nonspecifically into the lipid double layer of the viral envelope, which alters the fluidity of the membrane [132].

Many people consider herbal remedies to be harmless, but using them without medical advice may lead to harmful effects on health, and in other cases some treatments may be ineffective and thus the condition of a person with COVID-19 may worsen as a result of not being treated effectively.

There may be some effective herbal remedies in preventing and treating COVID-19 for some people, but the lack of information on the mechanism of effect, the appropriate dosage, side effects, toxicity and drug interactions makes them questionable, as they need more research and study. So far, there is no WHO-approved herbal remedy for COVID-19.

\section{Limitations}

There is insufficient information about the pharmacokinetic efficacy of the medicinal plant species in this study. These herbs that have been reported that have been used traditionally as adjuvant to relieve and treat symptoms of COVID-19, the effects of which remain unknown.

\section{Conclusion}

This research is working on studying some of the folk remedies that are widespread and naturally widespread in Syria. The importance of this research falls within the global efforts exerted to search for natural remedies that are cheap and effective to limit the spread of viral infections in general and in particular the emerging corona virus (COVID-19). The studied plants may have dual antiviral and immunomodulating effects, and thus can be adopted in preventing the spread of viral infection. Therefore, studies must be conducted on evaluating the effectiveness of Syrian medicinal plants rich in flavonoids and volatile oils as antiviral, especially the emerging corona virus, COVID-19.

\section{Declarations}

\section{Financial support (Funding)}

This study did not receive any particular gift from funding companies in the public, commercial, or not-for-profit sectors.

\section{Declaration of Competing interests}

We declare that there is no conflict of interest with any business organization concerning the study.

\section{Authors' Contributions}

C.H.: Data collection, methodology, investigation, compiled the literature sources, data analysis, evaluation, and interpretation, realization manuscript, helped in data, and made a substantial contribution to data analysis. A.N.: Evaluation the clinical data, performed data analysis and drafted the manuscript. M.I.H.A.: Review \& editing, designed the research and identification of plant species. All contributors understand and accepted the final document. 


\section{Acknowledgments}

The authors would like to express their gratitude to Mr. Besher Yazji, Mr. Fares Kallas, and Dr. M.Yasaar Abdeen president of Damascus University, and Dr. Jameela Hasian, Dr. Mays Khazem, Dr. Rasha El-Khateeb at Damascus University - Faculty of Pharmacy, Dr. Haifa Al-Ali, Dr. Aoula Moustapha at Al Baath University - Faculty of Pharmacy, Dr. Fawaz Shehneh, Dr. Adawia Kitaz at Aleppo University - faculty of Pharmacy, and Dr. Ahmad Karaali at Tishreen University - Higher Institute of Marine Research, and to Mr. Fawaz Saleh and Dr. Nasser Thallaj Mr. Hisham jabaji at Al Rasheed Private University, and Dr. Emad Kadi (God rest his soul), Dr. Abdel Aleem Bello, Dr. Bayan Tiba, Dr. Jalal Fandi the taxonomic specialists. Their thanks are also extended to The Syria Trust for Development (Mr. Shadi Alelshi, Mrs. Areen Al-Ali, Mr. Houssam Akel, Mr. Rafed Al-Ali) and the Syrian syndicate of pharmacists board and Dr. wafa kishi the head, the Syrian Scientific Society for Herbal Medicines and Complementary Medicines and Nutrition Chemist. Hisham Kasem for making this work possible. also they are thankful to the pharmacists and herbalists in this study for their valuable help in the documentation of indigenous ethnopharmacological knowledge.

\section{References}

1- World Health Organization (WHO): WHO traditional medicine strategy 2014-2023. [Internet]. 2013. Geneva. WHO Press [cited 2021 March 15]. Available from: https://apps.who.int/

2- Gurib-fakim A. 2006. Medicinal plants: Traditions of Yesterday and Drugs of Tomorrow. Molecular Aspects of Medicine. 27: $1-93$.

3- Houghton P. J. 1995. The Role of Plants in Traditional Medicine and Current Therapy. The Journal of Alternative and Complementary Medicine. 1(2), 131-143.

4- Baker D., Mocek U., Garr G. 2007. Natural Products Vs. Combinatorials: A Case Study, Natural Products - History, Diversity and Discovery. The Royal Society of Chemistry's. 66-72.

5- Roche J. et al. 2017. Traditional medicine sharing experiences from the field. ICHCAP \#HeritageAlive ichngoforum, Living Herit. Ser. UNESCO.

6- Weiss S.R., Leibowitz J.L. 2011. Coronavirus pathogenesis Advances in Virus Research. Academic Press Inc., p. 85-164

7- Xu X. et al. 2020. Evolution of the novel coronavirus from the ongoing Wuhan outbreak and modeling of its spike protein for risk of human transmission Sci. China Life Sci. 63 (3): p. 457-460, 10.1007/s11427-020-1637-5

8- World health Organization (WHO): . Novel Coronavirus (2019-nCoV) Situation Report-121 [Internet]. 2020. [cited 2021 March 15]. Available from: https://www.who.int/

9- Ji W., Wang W., Zhao X., Zai J., Li X. 2020. Cross-species transmission of the newly identified coronavirus 2019-nCoV, J. Med. Virol., 92 (4): p. 433-440, 10.1002/jmv.25682

10- WHO Director-General's opening remarks at the media briefing on COVID-19. [Internet]. 2020. [cited 2021 March 15]. Available from: https://www.who.int/

11- Centers for disease control and prevention (CDC), Symptoms of Coronavirus [Internet]. 2020. [cited 2021 March 15]. Available from: https://www.cdc.gov/

12- Becker K. H., Schoenbach K. H., Eden J. G. 2006. Microplasmas and applications. J. Phys. D. Appl. Phys. 39(3): 15-20.

13- Schneider W. M., Chevillotte M. D., Rice C. M. 2014. Interferon-Stimulated Genes: A Complex Web of Host Defenses. Annu. Rev. Immunol. 32: 513-545. 
14- Perrin L., Telenti A. 1998. HIV Treatment Failure: Testing for HIV Resistance in Clinical Practice. Science. 80(280): 18711873.

15- Revuelta-Herrero J. L. et al. 2018. Effectiveness, Safety, and Costs of a Treatment Switch to Dolutegravir Plus Rilpivirine Dual Therapy in Treatment-Experienced HIV Patients. Ann. Pharmacother. 52: 11-18.

16- [Internet]. 2020. [cited 2021 March 15]. Available from: http://countrystudies.us/syria/19.htm

17- Chaachouaya N., Douirab A., Zidaneb L. 2021. COVID-19, prevention and treatment with herbal medicine in the herbal markets of Salé Prefecture, North-Western Morocco, European Journal of Integrative Medicine. Volume 42.

18- Tenté A., Lougbégnon O., Houessou G., Dossou M., Codjia J. 2012. Etude ethnobotanique des ressources forestières ligneuses de la forêt marécageuse d'Agonvè et terroirs connexes au Bénin Tropicultura. 30 (1): p. 41-48.

19- Tabuti J.R.S., Lye K.A., Dhillion S.S. 2003. Traditional herbal drugs of Bulamogi, Uganda: Plants, use and administration J. Ethnopharmacol., 88 (1): p. 19-44, 10.1016/S0378-8741(03)00161-2

20- Yaseen G. et al. 2015. Ethnobotany of medicinal plants in the Thar Desert (Sindh) of Pakistan, J. Ethnopharmacol. 163: p. 43-59, 10.1016/j.jep.2014.12.053

21- Montvale NJ. 2000. PDR for Herbal Medicines. 2nd ed. [place unknown]: Medical Economics Company.

22- Reichling, J. et al. 2009. Antiviral activity of Rhus aromatica (fragrant sumac) extract against two types of herpes simplex viruses in cell culture. Die Pharmazie-An International Journal of Pharmaceutical Sciences 64(8): 538-541.

23- Gitanjali N. 2012. Antimicrobial Activity, Cytotoxicity and Phytochemical Analyses of Rhus aromatica, Rhus glabra and Sanguinaria canadensis Native to South Dakota. [South Dakota]: Electronic Theses and Dissertations. 1355.

24- Gülçın İ. et al. 2003. Screening of antioxidant and antimicrobial activities of anise (Pimpinella anisum L.) seed extracts. Food chemistry 83(3): 371-382.

25- Lee JB., Yamagishi C., Hayashi K., Hayashi T. 2011. Antiviral and immunostimulating effects of lignin-carbohydrate-protein complexes from Pimpinella anisum. Biosci Biotechnol Biochem. 75(3):459-65.

26- Shojaii A., Mehri A.F. 2012. Review of pharmacological properties and chemical constituents of Pimpinella anisum. International Scholarly Research Notices. 2012.

27- Eun-Mi C., Hwang J.K. 2004. Antiinflammatory, analgesic and antioxidant activities of the fruit of Foeniculum vulgare. Fitoterapia 75(6): 557-565.

28- Münir O., Gülçin İ., Küfrevioğlu Ö.l. 2003. Determination of in vitro antioxidant activity of fennel (Foeniculum vulgare) seed extracts. LWT-Food Science and Technology. 36(2): 263-271.

29- Shukla H.S., Dubey P., Chaturvedi R.V. 1989. Antiviral properties of essential oils of Foeniculum vulgare and Pimpinella anisum L. Agronomie 9(3): 277-279.

30- Maryam A. et al. 2019. Analysis and evaluation of the antimicrobial and anticancer activities of the essential oil isolated from Foeniculum vulgare from Hamedan, Iran. Natural product research 33(11): 1629-1632.

31- Matasyoh J.C. et al. 2009. Chemical composition and antimicrobial activity of the essential oil of Coriandrum sativum. Food Chemistry 113(2): 526-529.

32- Asres K. et al. 2001. Antiviral activity against human immunodeficiency virus type 1 (HIV-1) and type 2 (HIV-2) of ethnobotanically selected Ethiopian medicinal plants. Phytotherapy Research: An International Journal Devoted to Pharmacological and Toxicological Evaluation of Natural Product Derivatives 15(1): 62-69.

Page $14 / 21$ 
33- Melo D.A., Enayde, Filho J.M., Guerra N.B. 2005. Characterization of antioxidant compounds in aqueous coriander extract (Coriandrum sativum L.). LWT-Food Science and Technology 38(1): 15-19.

34- Rosana F. et al. 2000. Antioxidant activity of Ilex paraguariensis and related species. Nutrition research 20(10): 1437-1446.

35- Martin J., Prado G., et al. 2013. Antimicrobial activity of yerba mate (Ilex paraguariensis St. Hil.) against food pathogens. Revista Argentina de microbiologia 45(2): 93-98.

36- Lückemeyer D. et al. 2012. Effects of Ilex paraguariensis A. St. Hil.(yerba mate) on herpes simplex virus types 1 and 2 replication." Phytotherapy Research 26(4): 535-540.

37- Mejía d., González E., et al. 2010. Yerba mate tea (Ilex paraguariensis): Phenolics, antioxidant capacity and in vitro inhibition of colon cancer cell proliferation. Journal of Functional Foods 2(1): 23-34.

38- Karina K., Suntres Z.E. 2016. The antimicrobial properties of ginseng and ginseng extracts. Expert review of anti-infective therapy 14(1): 81-94.

39- Lee J.S. et al. 2014. Antiviral activity of ginseng extract against respiratory syncytial virus infection." International journal of molecular medicine 34(1): 183-190.

40- Kim H.G. et al. 2011. Antioxidant effects of Panax ginseng CA Meyer in healthy subjects: a randomized, placebo-controlled clinical trial. Food and Chemical Toxicology 49(9): 2229-2235.

41- Lee W.S., Rhee D.K. 2021. Corona-Cov-2 (COVID-19) and ginseng: Comparison of possible use in COVID-19 and influenza. J Ginseng Res.

42- Asdadi A. et al. 2020. Chemical characterization of essential oil of Artemisia herba-alba asso and his possible potential against covid-19. Journal of Analytical Sciences and Applied Biotechnology 2(2): 2-2.

43- Shawky E., Nada A., Ibrahim R. 2020. Potential role of medicinal plants and their constituents in the mitigation of SARS-CoV2: identifying related therapeutic targets using network pharmacology and molecular docking analyses. RSC Advances 10(47): 27961-27983.

44- Mohamed, Abou El-Hamd H., et al. 2010. Chemical constituents and biological activities of Artemisia herba-alba. Records of Natural Products 4(1): 1-25.

45- Yashphe J. et al. 1979. Antibacterial activity of Artemisia herba-alba. Journal of pharmaceutical sciences 68(7): $924-925$.

46- Younsi F. et al. 2016. Essential oil and phenolic compounds of Artemisia herba-alba (Asso.): Composition, antioxidant, antiacetylcholinesterase, and antibacterial activities." International journal of food properties. 19(7): 1425-1438.

47- Roby M.H. et al. 2013. Antioxidant and antimicrobial activities of essential oil and extracts of fennel (Foeniculum vulgare L.) and chamomile (Matricaria chamomilla L.)." Industrial crops and products. 44: 437-445.

48- Mohammad S.M. 2011. Study on Cammomile (Matricaria chamomilla L.) usage and Farming." Advances in Environmental Biology 5(7): 1446-1453.

49- Stanojevic L.P. et al. 2016. Chemical composition, antioxidant and antimicrobial activity of chamomile flowers essential oil (Matricaria chamomilla L.). Journal of essential oil bearing plants. 19(8): 2017-2028.

50- Romeilah R.M. 2009. Anticancer and antioxidant activities of Matricaria chamomilla L. and Marjorana hortensis essential oils. Research journal of medicine and medical sciences. 4(2): 332-339.

51- Nirmala P., Selvaraj T. 2011. Anti-inflammatory and anti-bacterial activities of Glycyrrhiza glabra L. Journal of Agricultural Technology. 7(3): 815-23. 
52- Martins N. et al. 2015. Characterization of phenolic compounds and antioxidant properties of Glycyrrhiza glabra L. rhizomes and roots. RSC Advances. 5(34): 26991-26997.

53 Asl N., Hosseinzadeh H. 2007. Review of antiviral effects of Glycyrrhiza glabra L. and its active component, glycyrrhizin. Journal of Medicinal Plants. 6(22): 1-12.

54- Allahverdiyev A. et al. 2004. Antiviral activity of the volatile oils of Melissa officinalis L. against Herpes simplex virus type-2. Phytomedicine. 11(7-8): 657-661.

55- Cohen, R.A., Louis S.K., Ernest Jr. 1964. Antiviral Activity of Melissa officinalis (Lemon Balm) Extract." Proceedings of the Society for Experimental Biology and Medicine 117(2): 431-434.

56- Mimica-Dukic N. et al. 2004. Antimicrobial and antioxidant activities of Melissa officinalis L.(Lamiaceae) essential oil." Journal of agricultural and food chemistry 52(9): 2485-2489.

57- Hamza A. et al. 2016. Melissa officinalis protects against doxorubicin-induced cardiotoxicity in rats and potentiates its anticancer activity on MCF-7 cells. PloS one 11(11): e0167049.

58- Sharma V. et al. 2014. In vitro anticancer activity of extracts of Mentha spp. against human cancer cells. Indian Journal of Biochemistry \& Biophysics. 51(5):416-419.

59- Hajlaoui H. et al. 2009. Biological activities of the essential oils and methanol extract of tow cultivated mint species (Mentha longifolia and Mentha pulegium) used in the Tunisian folkloric medicine." World Journal of Microbiology and Biotechnology. 25(12): 2227-2238.

60- Teixeira B. et al. 2012. European pennyroyal (Mentha pulegium) from Portugal: Chemical composition of essential oil and antioxidant and antimicrobial properties of extracts and essential oil. Industrial Crops and Products. 36(1): 81-87.

61- Formisano Ca. et al. 2014. Chemical composition of essential oils and in vitro antioxidant properties of extracts and essential oils of Calamintha origanifolia and Micromeria myrtifolia, two Lamiaceae from the Lebanon flora." Industrial Crops and Products. 62: 405-411.

62- Al-Samarraei K.W. et al. 2011. Cytogenetic and Cytotoxic study of Micromeria myrtifolia Extract on Animal and Human Cancer Cell Line. Iraq Journal of Market Research and Consumer Protection. 3(6): 131-144.

63- Küpeli A.E., Dereli F.G., Ilhan M. 2019. Assessment of antidepressant effect of the aerial parts of micromeria myrtifolia Boiss. \& Hohen on mice. Molecules. 24(10): 1869.

64- Shamseddine L., Jose J.C. 2020. Composition's effect of Origanum Syriacum essential oils in the antimicrobial activities for the treatment of denture stomatitis. Odontology. 109: 327-335.

65- Rakover Y., Eran B.A., Goldstein L.H. 2008. The treatment of respiratory ailments with essential oils of some aromatic medicinal plants. Harefuah. 147(10): 783-8.

66- Matza-Porges S. et al. 2014. A new antiviral screening method that simultaneously detects viral replication, cell viability, and cell toxicity. Journal of virological methods. 208: 138-143.

67- Alma M.H. et al. 2003. Screening chemical composition and in vitro antioxidant and antimicrobial activities of the essential oils from Origanum syriacum L. growing in Turkey. Biological and Pharmaceutical Bulletin. 26(12): 1725-1729.

68- Bozin B. et al. 2007. Antimicrobial and antioxidant properties of rosemary and sage (Rosmarinus officinalis L. and Salvia officinalis L., Lamiaceae) essential oils. Journal of agricultural and food chemistry. 55(19): 7879-7885. 
69- Zaouali, Y., Taroub B., Mohamed B. 2010. Essential oils composition in two Rosmarinus officinalis L. varieties and incidence for antimicrobial and antioxidant activities. Food and Chemical Toxicology. 48(11): 3144-3152.

70- Nasr-Eldin M.A., Ahmed A., Dina B. 2017. Antibiofilm and antiviral potential of leaf extracts from Moringa oleifera and rosemary (Rosmarinus officinalis Lam.). Egyptian Journal of Microbiology. 52(1): 129-139.

71- Al-Mariri A. et al. 2013. Antibacterial activity of Thymus syriacus boiss essential oil and its components against some Syrian gram-negative bacteria isolates." Iranian journal of medical sciences. 38(2): 180.

72- Al-Mariri A. et al. 2013. Antibacterial activity of Thymus syriacus boiss essential oil and its components against some Syrian gram-negative bacteria isolates. Iranian journal of medical sciences. 38(2): 180.

73- Jamil D.M. 2009. Characterisation, Antioxidant and Antimicrobial Activity of Thymus syriacus Boiss. var. syriacus and Thymbra spicata L. from Kurdistan-Iraq. University of Surrey (United Kingdom).

74- Yang C.H., Li R.X., Chuang L.Y. 2012. Antioxidant activity of various parts of Cinnamomum cassia extracted with different extraction methods. Molecules. 17(6): 7294-7304

75- Huang D.F. et al. 2014. Chemical constituents, antibacterial activity and mechanism of action of the essential oil from Cinnamomum cassia bark against four food-related bacteria. Microbiology. 83(4): 357-365.

76- Clark M. "New open patent for COVID-19 treatment utilizing NAC and Cinamomum Cassia filed. Los Angeles, CA., September 7, 2020-Orange Orchid Research files open, provisional patent on SARS-CoV-2/COVID-19 treatment with the United States Patent and Trademark Office (USPTO).

77- Parmar, G. 2020. A Paradigm Shift of Herbal Remedies in SARS-COVID-19 Pandemic. Journal of Integrated Health Sciences. 8(2): 55-56.

78- Wilkin P.J. et al. 2020. The Undiscovered Potential of Essential Oils for Treating SARS-CoV-2 (COVID-19). Current Pharmaceutical Design. 26(41): 5261-5277.

79- Fitriani I.N. et al. 2020. In Silico Approach of Potential Phytochemical Inhibitor from Moringa oleifera, Cocos nucifera, Allium cepa, Psidium guajava, and Eucalyptus globulus for the treatment of COVID-19 by Molecular Docking. Research Square. 1-25.

80- Damjanović-Vratnica B. et al. 2011. Antimicrobial effect of essential oil isolated from Eucalyptus globulus Labill. from Montenegro. Czech Journal of Food Sciences. 29(3): 277-284.

81- Vázquez G. et al. 2008. Antioxidant activity and phenolic content of chestnut (Castanea sativa) shell and eucalyptus (Eucalyptus globulus) bark extracts. Industrial crops and products 28(3): 279-285.

82- Romani A. et al. 2004. Evaluation of antioxidant effect of different extracts of Myrtus communis L. Free radical research. 38(1): 97-103.

83- Azimi M., Hasheminasab F.S. 2020. Evaluating the efficacy and safety of the myrtle (Myrtus communis) in treatment and prognosis of patients suspected to novel coronavirus disease (COVID-19): study protocol for a randomized controlled trial. Trials. 21(1): 1-5.

84- Aleksic V., Petar K. 2014. Antimicrobial and antioxidative activity of extracts and essential oils of Myrtus communis L. Microbiological research. 169(4): 240-254.

85- Romani A. et al. 2004. Evaluation of antioxidant effect of different extracts of Myrtus communis L. Free radical research. 38(1): 97-103. 
86- Chaieb K. et al. 2007. Antioxidant properties of the essential oil of Eugenia caryophyllata and its antifungal activity against a large number of clinical Candida species. Mycoses. 50(5): 403-406.

87- Öztürk H. 2005. The anti-inflammatory activity of Eugenia caryophyllata essential oil: an animal model of anti-inflammatory activity. European Journal of General Medicine. 2(4).

88- Yadav M.K. et al. 2013. Antimicrobial activities of Eugenia caryophyllata extract and its major chemical constituent eugenol against Streptococcus pneumoniae. Apmis. 121(12): 1198-1206.

89- Silva S. et al. 2006. Phenolic compounds and antioxidant activity of Olea europaea L. fruits and leaves. Food Science and Technology International. 12(5): 385-395.

90- Salih R.H. et al. 2017. Antiviral effects of olea europaea leaves extract and interferon-beta on gene expression of newcastle disease virus. Adv Anim Vet Sci. 5(11): 436-45.

91- Pereira A.P. et al. 2007. Phenolic compounds and antimicrobial activity of olive (Olea europaea L. Cv. Cobrançosa) leaves. Molecules. 12(5): 1153-1162.

92- Bintang M., Pasaribu F.H., Safira U.M., Sidhartha T. 2018. Identification of bioactive compound from Nigella sativa, Allium sativum, propolis and Oleaeuropaea mixture as antibacterial and antifungal agent. IOP Conf. Ser.: Earth Environ. Sci. 196 012041

93- Ghannadi A., Valiollah H., Hadi J. 2005. An investigation of the analgesic and anti-inflammatory effects of Nigella sativa seed polyphenols. Journal of medicinal food. 8(4): 488-493.

94- Umar S. et al. 2016. Protective and antiviral activities of Nigella sativa against avian influenza (H9N2) in turkeys. Journal of the Saudi Society of Agricultural Sciences. 10.

95- Salman, M.T., Rahat A.K., Indu S. 2008. Antimicrobial activity of Nigella sativa Linn. seed oilagainst multi-drug resistant bacteria from clinical isolates. Natural Product Radiance. 7(1).

96- Schieber A., Mihalev K., Berardini N., Mollov P., Carle R. 2005. Flavonol Glycosides from Distilled Petals of Rosa damascena Mill. Zeitschrift für Naturforschung. 60(5-6): 379-384.

97- Naquvi K.J., Ansari S.H., Ali M., Najmi A.K. 2014. Volatile oil composition of Rosa damascena Mill. (Rosaceae). Journal of Pharmacognosy and Phytochemistry. 2(5): 177-181.

98- Shokouhinejad N. et al. 2010. Antimicrobial effect of Rosa damascena extract on selected endodontic pathogens. Journal of the California Dental Association. 38(2): 123-126.

99- Thallaj, N. et al. 2020. Evaluation of Antimicrobial Activities and Bioactive Compounds of Different Extracts Related to Syrian Traditional Products of Damask Rose (Rosa damascena). Open Access Library Journal. 7(5):1-21.

100- Özkan G. et al. 2004. Note: Antioxidant and antibacterial activities of Rosa damascena flower extracts. Food Science and Technology International. 10(4): 277-281.

101- Hajhashemi V., Ghannadi A., Hajiloo M. 2010. Analgesic and anti-inflammatory effects of Rosa damascena hydroalcoholic extract and its essential oil in animal models. Iranian journal of pharmaceutical research: IJPR. 9(2): 163.

102- Amorim J.L. et al. 2016. Anti-inflammatory properties and chemical characterization of the essential oils of four citrus species. PloS One. 11(4): e0153643.

103- Huang Y.S., Ho S.C. 2010. Polymethoxy flavones are responsible for the anti-inflammatory activity of citrus fruit peel. Food Chemistry. 119(3): 868-873.

Page 18/21 
104- Moosavy M.H. et al. 2017. Antioxidant and antimicrobial activities of essential oil of Lemon (Citrus limon) peel in vitro and in a food model. Journal of food quality and hazards control 4(2): 42-48.

105- Gebara R. et al. 2020. Identification and Characterization of Two Defensins from Capsicum annuum Fruits that Exhibit Antimicrobial Activity. Probiotics and antimicrobial proteins. 12(3): 1253-1265.

106- Materska M., Perucka I. 2005. Antioxidant activity of the main phenolic compounds isolated from hot pepper fruit (Capsicum annuum L.). Journal of Agricultural and food Chemistry. 53(5): 1750-1756.

107- Hernández-Ortega M. et al. 2012. Antioxidant, antinociceptive, and anti-inflammatory effects of carotenoids extracted from dried pepper (Capsicum annuum L.). Journal of Biomedicine and Biotechnology. 2012.

108- Bagad A.S. et al. 2013. Comparative evaluation of anti-inflammatory activity of curcuminoids, turmerones, and aqueous extract of Curcuma longa. Advances in pharmacological sciences. 2013.

109- Maizura M., Aminah A., Wan Aida W.M. 2011. Total phenolic content and antioxidant activity of kesum (Polygonum minus), ginger (Zingiber officinale) and turmeric (Curcuma longa) extract. International Food Research Journal. 18(2).

110- Niamsa N., Sittiwet C. 2009. Antimicrobial activity of Curcuma longa aqueous extract. Journal of Pharmacology and Toxicology. 4(4): 173-177.

111- Ikpeama, A., Onwuka G.I., Nwankwo C. 2014. Nutritional composition of Tumeric (Curcuma longa) and its antimicrobial properties. International Journal of Scientific and Engineering Research. 5(10): 1085-1089.

112-Sasidharan I., Menon A.N. 2010. Comparative chemical composition and antimicrobial activity fresh \& dry ginger oils (Zingiber officinale Roscoe). International Journal of Current Pharmaceutical Research 2(4): 40-43.

85- Stoilova I. et al. 2007. Antioxidant activity of a ginger extract (Zingiber officinale). Food chemistry. 102(3): 764-770.

113- Thomson M. et al. 2002. The use of ginger (Zingiber officinale Rosc.) as a potential anti-inflammatory and antithrombotic agent. Prostaglandins, leukotrienes and essential fatty acids. 67(6): 475-478.

114- Habib S.M. et al. 2008. Ginger extract (Zingiber officinale) has anti-cancer and anti-inflammatory effects on ethionineinduced hepatoma rats. Clinics. 63(6): 807-813.

115- Kaushik S. et al. 2020. Anti-viral activity of Zingiber officinale (Ginger) ingredients against the Chikungunya virus. Virusdisease. 31(3): 270-276.

116- Ahkam A.H. et al. 2020. Virtual prediction of antiviral potential of ginger (Zingiber officinale) bioactive compounds against spike and MPro of SARS-CoV2 protein. Berkala Penelitian Hayati. 25(2): 52-57.

117- Carmona M., Llorach R., Obon C., Rivera D. 2005. Zahraa, a Unani multicomponent herbal tea widely consumed in Syria: components of drug mixtures and alleged medicinal properties. Journal of Ethnopharmacology. 102: 344-350.

118- Che C., George V., ljinu T., Pushpangadan P., Andrae-Marobela K. 2017. Traditional medicine: pharmacognosy. [place unknown]: Academic Press. p.15.

119- International Bioethics Committee (IBC): Report of the IBC on traditional medicine systems and their ethical implications. [Internet]. 2013. Paris: UNESCO, [cited 2021 March 15]. Available from: http://unesdoc.unesco.org/

120- Ghosh D. 2005. Anthocyanins and Anthocyanin-Rich Extracts in Biology and Medicine: Biochemical, Cellular, and Medicinal Properties. Curr. Top. Nutraceutical Res. 3: 113-124.

121- Duthie G.G., Duthie S. J., Kyle J.M. 2000. Plant Polyphenols In Cancer and Heart Disease: Implications as Nutritional Antioxidants. Nutr Res Rev. 13(1):79-106.

Page 19/21 
122- Amin F. U., Shah S. A., Badshah H., Khan M., Kim M. O., 2017. Anthocyanins Encapsulated by PLGA/PEG Nanoparticles Potentially Improved its Free Radical Scavenging Capabilities Via P38/JNK Pathway against Aß1-42-Induced Oxidative Stress. J. Nanobiotechnology. 15: 1-16.

123- Pour P. M., Fakhri S., Asgary S., Farzaei M.H., Echeverría J. 2019. The signaling pathways, and therapeutic targets of antiviral agents: Focusing on the Antiviral Approaches and Clinical Perspectives of Anthocyanins in the Management of Viral Diseases. Front. Pharmacol. 10 (1207): 1-23.

124- Nowakowska Z. 2007. A review of anti-infective and anti-inflammatory chalcones, Eur. J. Med. Chem. 42(2): 125-137. 125- Chávez J.H. et al. 2006. Evaluation of antiviral activity of phenolic compounds and derivatives against rabies virus. Vet. Microbiol. 116(1-3): 53-59.

126- Yang Y., Islam M.S., Wang J., Li Y., Chen X. 2020. Traditional Chinese medicine in the treatment of patients infected with 2019-new coronavirus (SARS-CoV-2): a review and perspective. Int. J. Biol. Sci. 16(10): 1708-1717.

127- Khaerunnisa S., Kurniawan H., Awaluddin R., Suhartati S. 2020. Potential inhibitor of COVID-19 main protease (M $\left.{ }^{\text {pro }}\right)$ from Several medicinal plant compounds by molecular docking study. Preprints. 1: 1-14.

128- Park J.Y. et al. 2017. Evaluation of polyphenols from Broussonetia papyrifera as coronavirus protease inhibitors. J. Enzyme Inhib. Med. Chem. 32(1): 504-512.

129- Wink M. 2020. Potential of DNA intercalating alkaloids and other plant secondary metabolites against SARS-CoV-2 causing COVID-19. Diversity. 12(5): 1-10.

130- Dhama K. et al. 2018. Medicinal and therapeutic potential of herbs and plant metabolites / extracts countering viral pathogens - current knowledge and future prospects. Curr. Drug Metab. 19(3): 236-263.

131- Akram M. et al. 2018. Antiviral potential of medicinal plants against HIV, HSV, influenza, hepatitis, and coxsackievirus: a systematic review, Phytother. Res. 32(5): 811-822.

132- Ben-Shabat S., Yarmolinsky L., Porat D., Dahan A. 2020. Antiviral effect of phytochemicals from medicinal plants: Aapplications and drug delivery strategies. Drug Deliv. Transl. Res. 10(2): 354-367.

133- Sharma A.D., Kaur I. 2020. Molecular docking studies on Jensenone from eucalyptus essential oil as a potential inhibitor of COVID 19 corona virus infection. Research and reviews in biotechnology and biosciences. 2(7):59-66.

\section{Figures}




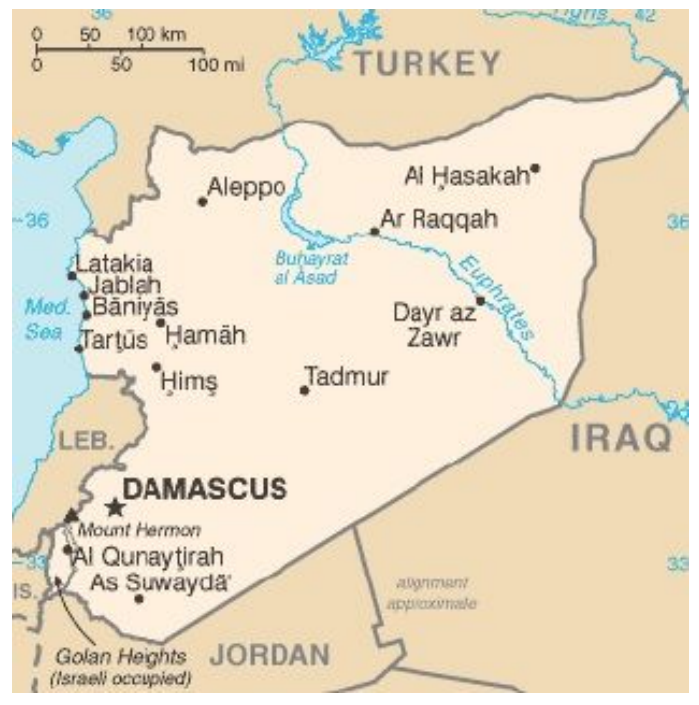

\section{Figure 1}

Geographical Location of the study area, Syria [16]. Note: The designations employed and the presentation of the material on this map do not imply the expression of any opinion whatsoever on the part of Research Square concerning the legal status of any country, territory, city or area or of its authorities, or concerning the delimitation of its frontiers or boundaries. This map has been provided by the authors.

\section{Supplementary Files}

This is a list of supplementary files associated with this preprint. Click to download.

- AppendixA.pdf

- SupplementaryMaterial.docx 